\title{
Bayesian Modeling of Working Memory and Inhibitory Control
}

\author{
Héctor A. Cepeda-Freyre ${ }^{1}$, Gregorio Garcia-Aguilar ${ }^{1} \&$ J. Jacobo Oliveros-Oliveros ${ }^{2}$ \\ ${ }^{1}$ Benemerita Universidad Autonoma de Puebla, Faculty of Psychology, Mexico. \\ ${ }^{2}$ Benemerita Universidad Autonoma de Puebla, Faculty of Physics and Mathematic Sciences, Mexico \\ Correspondence: Gregorio Garcia-Aguilar, Benemerita Universidad Autonoma de Puebla, Faculty of Psychology, \\ Mexico. E-mail: gregorio.garcia@correo.buap.mx
}

Received: October 9, 2018

Accepted: October 30, 2018

Online Published: November 7, 2018

doi:10.5539/ijps.v10n4p53

URL: https://doi.org/10.5539/ijps.v10n4p53

\begin{abstract}
In cognitive science, working memory is a core cognitive ability that might be functionally related to other capacities, such as perceptual processes, inhibitory control, memory and attention processes and executive functions. The mathematical study of working memory has been explored before. However, there is not enough research aiming to study the relationship between working memory and inhibitory control. This is the objective of the present report. Bayesian hypothesis testing is often more robust than traditional p-value null hypothesis testing. Yet, the number of studies using this approach is still limited. A secondary objective of this paper is to contribute to fill that gap, as well as provide an empirical application of Bayesian hypothesis testing using cognitive and behavioral data. A within-subjects design was used to measure working memory function for three types of visual stimuli that varied in the degree of attentional interference they were designed to elicit. Data collected was contrasted with measurements of inhibitory control and analyzed using Bayes' theorem. Our results provide evidence against the theoretical relationship of working memory and inhibitory control. This outcome is analyzed in light of related cognitive research.
\end{abstract}

Keywords: working memory, inhibitory control, bayesian analysis

\section{Introduction}

Working memory is a cognitive process that has received the attention of a multitude of thinkers and researchers through the years (Cassel, Cassel, \& Manning, 2013). Working memory involves the capacity for maintaining and storing information for short periods of time. The information to maintain may come from visual and auditive sensory modalities mainly, but cognitive psychologist have interested in other forms of information (Baddeley, 2003). The concept of working memory has been invented by Miller, Galanter and Pribram, but the leading theorist is A. Baddeley (Baddeley, 2017).

Working memory is a type of memory used to process several cognitive operations. One of the models most used to describe it is the one proposed by Baddeley and Hitch in 1974 (Baddeley \& Hitch, 1974). The authors use empirical evidence to create a model that sees working memory as a dynamic system, a special kind of temporal buffer that both stores and processes information. In this model, there exist three components: a visuospatial sketchpad, dealing with visual information; a phonological loop in charge of the linguistic information; these two components are for storing information. A central executive component, working as a control system complete the model. Recently, a forth component, called episodic buffer has been added, as an interface between long term and working memory (Baddeley, 2003) .

The working memory (WM from now on) research has been driven by theory. At first, scientific discussion regarding memory focused on whether it was a single capacity or multiple ones; the first position saw memory as an unitary capacity that handled all tasks related to retention and retrieval of information, the second point of view theorized the existence of several types of memory, which were independent to each other and were divided according to the type of functions they carried out; evidence obtained through the years has supported this last posture (Atkinson \& Shiffrin, 1968; Repovš \& Baddeley, 2006)

However, because memory is a double storing and processing function cognitive capacity, it is not clear at the present how much of these functions, or in what degree memory works together with other cognitive capacities, hence facilitating the processing; or if memory is an independent and unrelated process, coming along with different cognitive processes, as attention or inhibitory control. For this reason, two different approximations 
exist when it comes to the study of WM (Baddeley, 1992): the first approximation tries to find the relations between WM and other cognitive capacities, the second approach tries to analyze the WM structure as a cognitive system.

Supporters of the first approximation state that information to be retained may come with irrelevant, distracting or conflicting information, hence a cognitive system for suppressing these is needed. This system has been identified as inhibitory control (Dempster, 1991). The debate is about what comes first, WM or inhibitory control? Are they related?

For A. Diamond, both WM and inhibitory control are interrelated executive functions. The statement of Diamond subordinates the cognitive maturation and development to inhibitory control however . For example, she quoted a longitudinal study by Moffitt, remarking the relevance of inhibitory control in cognitive development (Moffitt et al., 2011). Both cognitive processes are interrelated in early childhood -until 8 years-; however, but are separated during late childhood and early adolescence (Shing, Lindenberger, Diamond, Li, \& Davidson, 2010).

In the same vein, N. Duell and collaborators have studied the Stroop task in its inhibition component, by the manipulation of incongruent trials; and the WM by the low proportion of incongruent trials in a large sample of adolescents (Duell et al., 2018). The results prove a high correlation between inhibitory control accuracy and working memory; however, when using the response time, it seems that inhibitory control are affected by the memory load in adults.

This effect was reported in children by Diamond (Wright \& Diamond, 2014). If the WM load is kept constant, but variations in incongruent trials are made, hence children make more errors. This results are representative of the influence inhibitory control in accuracy of memory task during childhood.

Supporters of the second approximation take the initial concept of WM from Miller, Galanter and Pribram (Miller, Galanter, \& Pribram, 1960) arguing for WM to be a part of a more complex and extended process related to goals or Plans (Beaman, 2010). A second form of this approach argues in favor of an cognitive system from which WM emerges. This approximation conceives WM as dedicated system which is embedded into an extended cognitive structure (Cowan, 1988; 1999). This system has been identified as the executive attention (Engle, 2002).

We can show two interesting things about the second approximation. First, it seems that inhibitory control is a separable cognitive process, which do not influences WM. This is showed by the investigation of Borella, Carretti and De Beni (Borella, Carretti, \& De Beni, 2008). These authors assessed accuracy on WM task, both in visual and verbal modalities, as well as in verbal inhibitory tasks in adult subjects. Their first analysis aimed to correlate age with accuracy; the second a regression showed correlation between age and WM, meaning that this cognitive process decline with age. At the same time, inhibition do not have the same results, because inhibition correlates with age in older, but not in younger adults. Hierarchical analysis confirm the low rate influence of inhibition on memory.

In second place, WM is a general cognitive capacity, under which some components may be identified. Carriedo and collaborators (Carriedo, Corral, Montoro, Herrero, \& Rucian, 2016), showed differences in updating information (WM) until fifteen years old; inhibition showed changes until young adulthood however. Two main components emerged from this study: an active maintenance/suppression of information vs. a control of proactive interference. In the same vein, Redick and collaborators showed no relationship between WM and inhibition (Redick, Calvo, Gay, \& Engle, 2011). The WM capacity hence is related to the hypothesis of the differences of memory between individuals. The WM is part of an attentional account in which the maintenance and retrieval of information depends on the velocity of subjects' processing, but not merely on the interference-rich (inhibitory) of situations.

The present paper takes a posture in between both approximations. Using simple Bayesian modeling we explore the relationship between working memory and inhibitory control, and their interaction in relation to the presence of visual noise during a working memory task. We assessed subjects in order to identified high inhibitors from low ones. Then, we assessed the visual working memory (vWM from now on) in a three conditions paradigm.

\section{Methods and Materials}

\subsection{Sample}

Data analyzed in the present paper were collected from 30 undergraduate students, whom provided voluntary informed consent and authorized the usage of data obtained from their participation. The notation $S_{1}, S_{2}, \ldots, S_{n}$ will be used to refer to each one of the $n$ participants, while $S$ represents any random participant. Additionally, 
half of the participants are denoted $I_{H}$ (High inhibitory control), and the other half $I_{L}$ (Low inhibitory control). This denomination is assigned according to their scores on a "Go/No-no" task (Gomez \& Perea, 2007) and a median split of said scores (Iacobucci, Posavac, Kardes, Schneider, \& Popovich, 2015).

\subsection{Experimental Conditions}

In the present paper three stimulus types: a control and two test conditions, that vary in the degree of visual interference present were applied, for assessing visuospatial working memory (vWM). $\alpha, \beta, \gamma$, which represent the type of stimulus used. In condition $\alpha$ the stimuli used consisted of 4 black irregular polygons, without a background figure; this is the control condition. In condition $\beta$ the stimuli used consisted of 4 black irregular polygons, with a thick gray line forming the perimeter of a square. In condition $\gamma$ the stimuli were 4 black polygons as in the previous conditions, with a background figure consisting of an irregular polygon. (see figure $1)$.

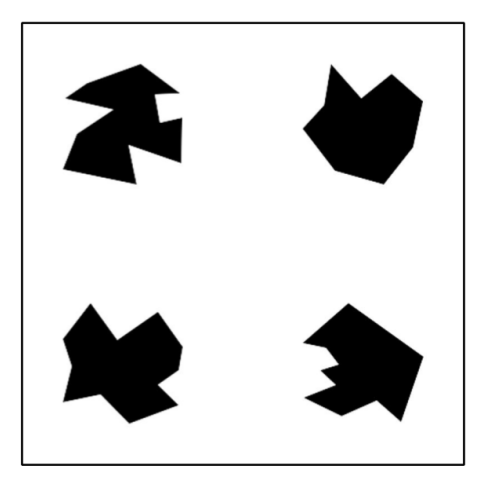

$\boldsymbol{\alpha}$

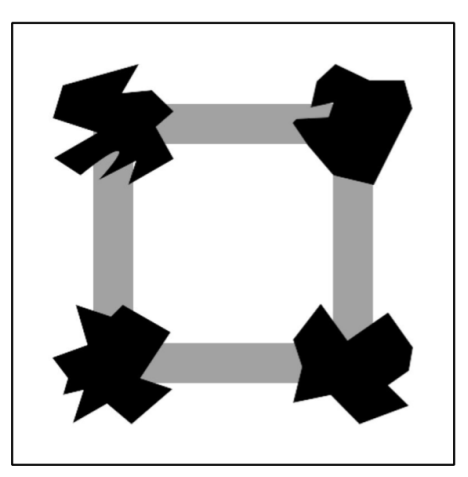

$\boldsymbol{\beta}$

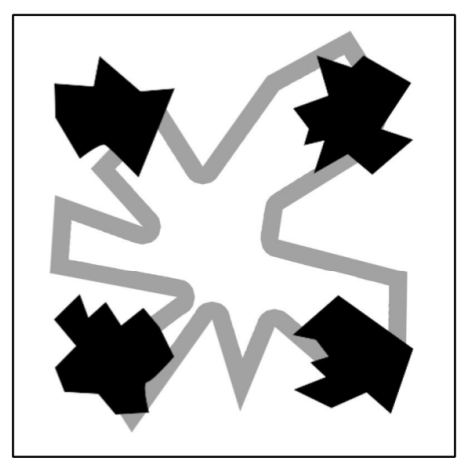

$\gamma$

Figure 1. Alpha, beta, and gamma experimental conditions.

\subsection{Experimental Blocks and Design}

An experimental block, denoted $B^{m}$ is a series of $k$ trials under an experimental condition $m \in\{\alpha, \beta, \gamma\}$. Each volunteer performed tasks in an experimental block of each condition, following a repeated measures design. Working memory measurements were obtained using a change detection task. One trial of this task is denoted $t$, and it belongs to an experimental block $B$.

$$
\begin{aligned}
& t \in B \\
& B \supset\left\{t_{1} \ldots t_{k}\right\}
\end{aligned}
$$

In the present paper $k=150$. Additionally, $B$ contains for every trial correct answers $V$ and responses given by the experimental subject, these responses are denoted by $U$. Finally, each experimental block $B$ contains a result $Q$ given by the combination of $V$ and $U$, as is shown in (8). It follows that:

$$
\begin{aligned}
& \forall t_{i} \in B, V_{i} \in B \\
& \forall t_{i} \in B, U_{i} \in B \\
& \forall t_{i} \in B, Q_{i} \in B \\
& \quad B \supset\left\{t_{1}, \ldots, t_{k}, V_{1}, \ldots, V_{k}, U_{1}, \ldots, U_{k}, Q_{i}, \ldots, Q_{k}\right\}
\end{aligned}
$$

In every $t$ within an experimental block $B$, a subject denoted by $S$ is shown an stimulus $E_{1}$ for 1.5 seconds on a computer screen, at the end of this period of time, the computer screen remains blank for another 1.5 seconds after which $S$ is shown a second stimulus $E_{2}$. $S$ must then produce a response $U . U_{i}=\Delta_{\theta}$ if $S$ declares that $\left\{\mathrm{E}_{1}=E_{2}\right\} \in t_{i}, U_{i}=\Delta$ if $S$ declares that $\left\{\mathrm{E}_{1} \neq E_{2}\right\} \in t_{i}$. Each $t_{i} \in B$ has a correct answer $V_{i}$, which is given by the equation:

$$
\left(\left\{E_{1}=E_{2}\right\} \in t_{i} \Rightarrow\left\{V_{i} \in t_{i}\right\}=\Delta_{\theta}\right) \vee\left(\left\{V_{i} \in t_{i}\right\}=\Delta\right)
$$

Each $t_{i}$ has a different result $Q_{i}$ depending on the combination of $V_{i}$ and $U_{i}$ : 


$$
\begin{array}{lll}
\left(V_{i}=\Delta_{\theta}\right. & \left.\& U_{i}=\Delta_{\theta}\right) & \Rightarrow Q_{i}=Q_{C R} \\
\left(V_{i}=\Delta\right. & \left.\& U_{i}=\Delta\right) & \Rightarrow Q_{i}=Q_{H} \\
\left(V_{i}=\Delta_{\theta}\right. & \left.\& U_{i}=\Delta\right) & \Rightarrow Q_{i}=Q_{F A} \\
\left(V_{i}=\Delta\right. & \left.\& U_{i}=\Delta_{\theta}\right) & \Rightarrow Q_{i}=Q_{M}
\end{array}
$$

Where $Q_{C R}$ denotes a correct rejection (lack of change detected), $Q_{H}$ denotes a hit (change identified), $Q_{M}$ denotes a miss (failed to detect a change that was present), and $Q_{F A}$ denotes a false alarm (change detected when it is not present). It follows that for each $t_{i} \in B$, there is a $Q_{i} \in B$. For each $j \in\{1 \ldots n\}$, a $S_{j}$ participates in an experimental block for each of the experimental conditions, in such way that for $S_{j}$ and index $m \in\{\alpha, \beta, \gamma\}$, the corresponding experimental block is denoted $B_{j}^{m}$. From the participation of $S_{j}$ in each $B_{j}^{m}$ the number of correct responses is obtained, this amount is denoted as $R_{j}^{m}$, and it is calculated as:

$$
\begin{aligned}
{[P]=} & \begin{cases}1 & \text { if } P \text { is correct } \\
0 & \text { if } P \text { is not correct }\end{cases} \\
& C(x, y, z)=\sum_{i=1}^{k}\left[Q_{i, y}^{z}=x\right] \\
& R_{j}^{m}=C\left(Q_{H}, j, m\right)+C\left(Q_{C R}, j, m\right)
\end{aligned}
$$

Where $Q_{i, j}^{m} \in B_{j}^{m}$.

Experimental blocks were presented to each subject following a balanced Latin square, as shown in Figure 2.

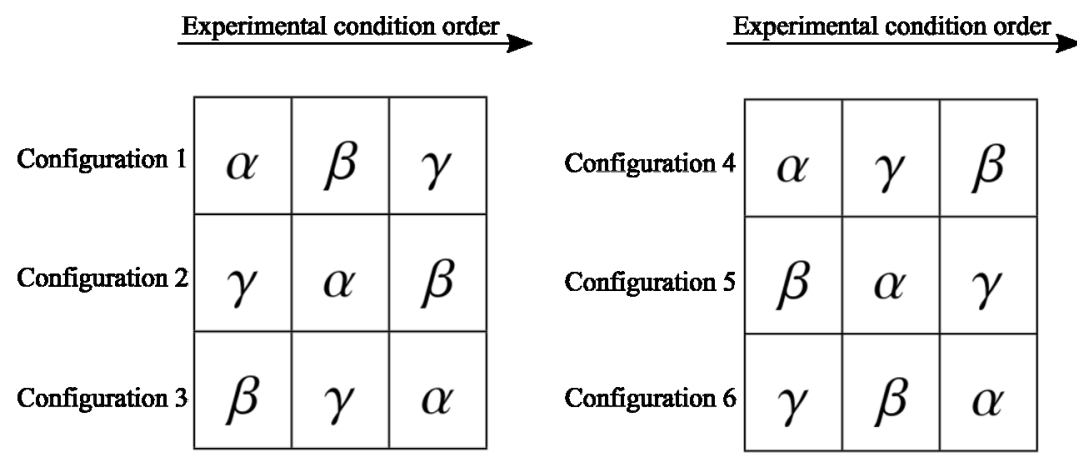

Figure 2. Latin square of the experimental design. Each row represents the chronological order of participation in each of the three conditions. There were 6 possible configurations in which a participant would perform the tasks in each block. Subjects were randomly assigned to one of the 6 configurations.

\subsection{Data Processing}

Data obtained from the experiment was analyzed using the statistical environment $\mathrm{R}$ (Team, 2011).

\subsection{Probabilistic Modeling}

Inhibitory control ability is denoted $I$, and every $S_{j}$ has their own correspondent $I_{j}$, where $j \in\{1,2, \ldots, n\}$. Using a median split, half of the $S_{j}$ are categorized has having high inhibitory control, in which case $I_{j}=I_{H}$; the other half is categorized as having low inhibitory control, in which case $I_{j}=I_{L}$. Inhibitory control can be then modeled as a Bernoulli variable, where $I_{H}=1$ and $I_{L}=0$, so for every experimental condition $m \in\{\alpha, \beta, \gamma\}$ there is a binomial distribution $x^{m} \sim B\left(n^{m}, p^{m}\right)$. Using the same notation from equation $9, n^{m}$ and $p^{m}$ are given by:

$$
\begin{gathered}
n^{m}=\sum_{j=1}^{n} R_{j}^{m} \\
r^{m}=\sum_{j=1}^{n} R_{j}^{m}\left[I_{j}=I_{H}\right] \\
p^{m}=\frac{r^{m}}{n^{m}}
\end{gathered}
$$

This same modeling style was used to evaluate the effect of the experimental conditions over working memory, 
defining a Bernoulli variable depending on the experimental condition from which a correct response was obtained, in such way that for $\beta$ a correct response under this condition will be coded as 1 while a correct response obtained under the $\alpha$ condition will be coded as 0 , resulting in a distribution $x_{\beta} \sim B\left(n_{\beta}, p_{\beta}\right)$. Regarding the $\gamma$ condition, the same logic can be applied resulting a distribution $x_{\gamma} \sim B\left(n_{\gamma}, p_{\gamma}\right)$.

$$
\begin{gathered}
n_{\beta}=\sum_{j=1}^{n} R_{j}^{\beta}+\sum_{j=1}^{n} R_{j}^{\alpha} \\
r_{\beta}=\sum_{j=1}^{n} R_{j}^{\beta} \\
p_{\beta}=\frac{r_{\beta}}{n_{\beta}} \\
n_{\gamma}=\sum_{j=1}^{n} R_{j}^{\gamma}+\sum_{j=1}^{n} R_{j}^{\alpha} \\
r_{\gamma}=\sum_{j=1}^{n} R_{j}^{\gamma} \\
p_{\gamma}=\frac{r_{\gamma}}{n_{\gamma}}
\end{gathered}
$$

\subsection{Bayesian Inference}

For each experimental condition $m \in\{\alpha, \beta, \gamma\}$ the posterior probability of 9 models was evaluated using Bayes' theorem (Stone, 2006):

$$
P(\text { Model } \mid \text { Data })=\frac{P(\text { Data } \mid \text { Model }) P(\text { Model })}{P(\text { Data })}
$$

$P$ (Model) represents the prior probability of each model. With respect to the evaluated models, these were created using binomial distributions $\sim B\left(\theta_{1}, \theta_{2}\right) . \quad P$ (Data $\mid$ Model $)$ was calculated using the equation:

$$
P(\text { Data } \mid \text { Model })=\left(\begin{array}{c}
\theta_{1} \\
r^{m}
\end{array}\right) \theta_{2}^{r^{m}}\left(1-\theta_{2}\right)^{\theta_{1}-r^{m}}
$$

where $r^{m}$ was calculated with equation (11). $P($ Data) is calculated with equation:

$$
P(\text { Data })=\sum_{q=1}^{9} P\left(\operatorname{Model}^{q}\right) P\left(\text { Data } \mid \operatorname{Model}^{q}\right)
$$

where $q$ indicates the model.

Three possible hypotheses were formulated for each experimental condition: The first hypothesis supposed both inhibitory control groups had the same probability of responding correctly to the task and was denoted $H_{\theta}$; the second hypothesis supposed $S_{j}$ had a larger probability of responding correctly to the task when $I_{j}=I_{H}$, this hypothesis was denoted $H_{1}$; lastly, the third hypothesis supposes $S_{j}$ has a larger probability of answering correctly to the task when $I_{j}=I_{L}$, this hypothesis was denoted $H_{2}$. For $H_{\theta}$ the parameter $\theta_{2}=0.5$ was evaluated. For $H_{1}$ the parameters $\theta_{2}=0.6, \theta_{2}=0.7, \theta_{2}=0.8$, and $\theta_{2}=0.9$ were evaluated. Finally, for $\mathrm{H}_{2}$ the parameters $\theta_{2}=0.4, \theta_{2}=0.3, \theta_{2}=0.2$, and $\theta_{2}=0.1$ were evaluated.

For the prior probability the same weight was assigned to two possibilities:

1.- There is an effect of inhibitory control $\left(H_{1}+H_{2}\right), \mathrm{p}=0.5$.

2.- There is no effect of inhibitory control $\left(H_{\theta}\right), \mathrm{p}=0.5$.

With respect to possibility number 1 , the same probabilistic weight was given to both hypotheses, in such way that $P\left(H_{1}\right)=0.25$ and $P\left(H_{2}\right)=0.25$, Meanwhile, each model was assigned an equal proportion of its corresponding hypothesis $(\mathrm{p}=0.0625)$. The prior probability of each of the nine models can be observed in Table 1. 
Table 1. Prior probability for the 9 models.

\begin{tabular}{l|l}
\hline Model's $\theta_{2}$ & Probability \\
\hline 0.1 & 0.0625 \\
\hline .2 & 0.0625 \\
\hline .3 & 0.0625 \\
\hline .4 & 0.0625 \\
\hline .5 & 0.5 \\
\hline .6 & 0.0625 \\
\hline .7 & 0.0625 \\
\hline .8 & 0.0625 \\
\hline .9 & 0.0625 \\
\hline
\end{tabular}

Note. Each column represents one of the 9 values used as parameter $\theta_{2}$ for the models, shown in the first row. Second row contains the prior for each model.

The same kind of inference was used to evaluate the effect of the experimental conditions where $H_{\theta}$ represents the absence of an effect of the experimental manipulation. $H_{1}$ indicates a positive effect of the manipulation over working memory, and $H_{2}$ indicates a negative effect.

\section{Results}

For condition $\alpha$, the resulting distribution $x^{\alpha} \sim B\left(n^{\alpha}, p^{\alpha}\right)$ took the following parameters: $n^{\alpha}=2387$, $p^{\alpha}=0.5081693$

The model with the highest posterior probability was $\theta_{2}=0.5$, with a probability of $p \approx 1$, Meanwhile, the rest of the models had an exceptionally small probability. These probabilities are shown in figure 5 , both in their real scale (A) and logarithmic scale (B).

Under condition $\beta$, the resulting distribution $x^{\beta} \sim B\left(n^{\beta}, p^{\beta}\right)$ acquired the following parameters: $n^{\beta}=2343$, $p^{\beta}=0.529236$.

The model with the highest posterior probability was also $\theta_{2}=0.5$, with $p \approx 1$. The probabilities of the different models are shown in Figure 6 (A), with their natural logarithm shown in (B).

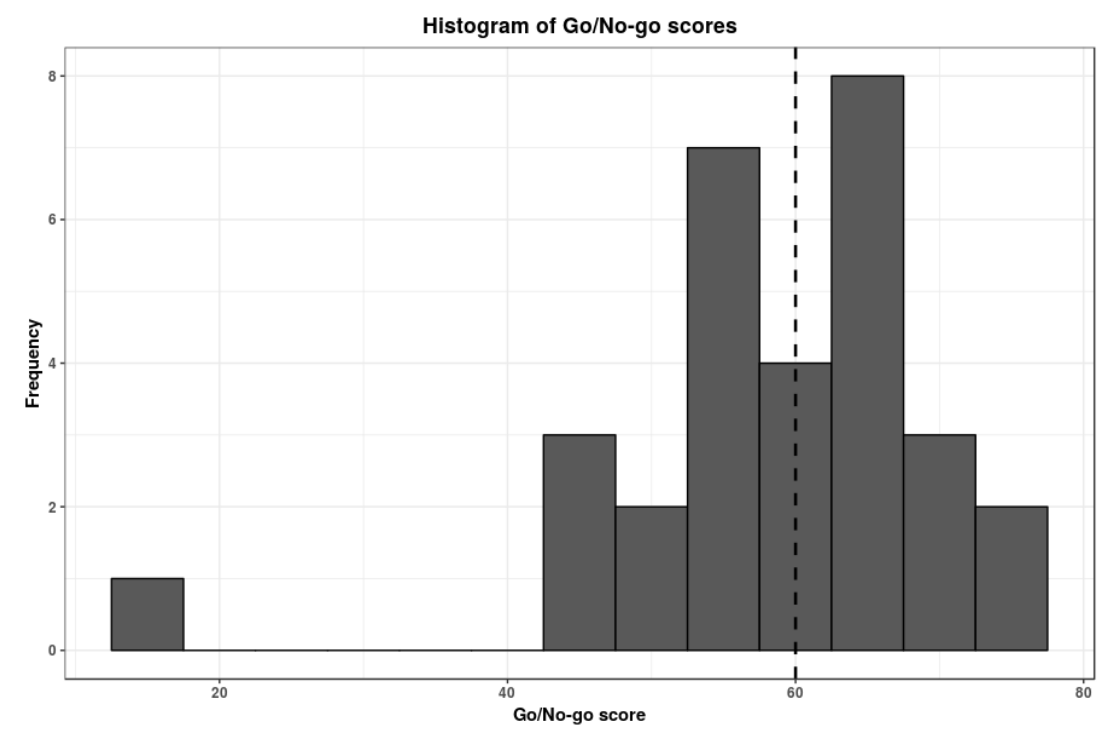

Figure 3. Histogram of scores for the Go/No-Go task.

Vertical dashed line indicates the score threshold for the median split. 


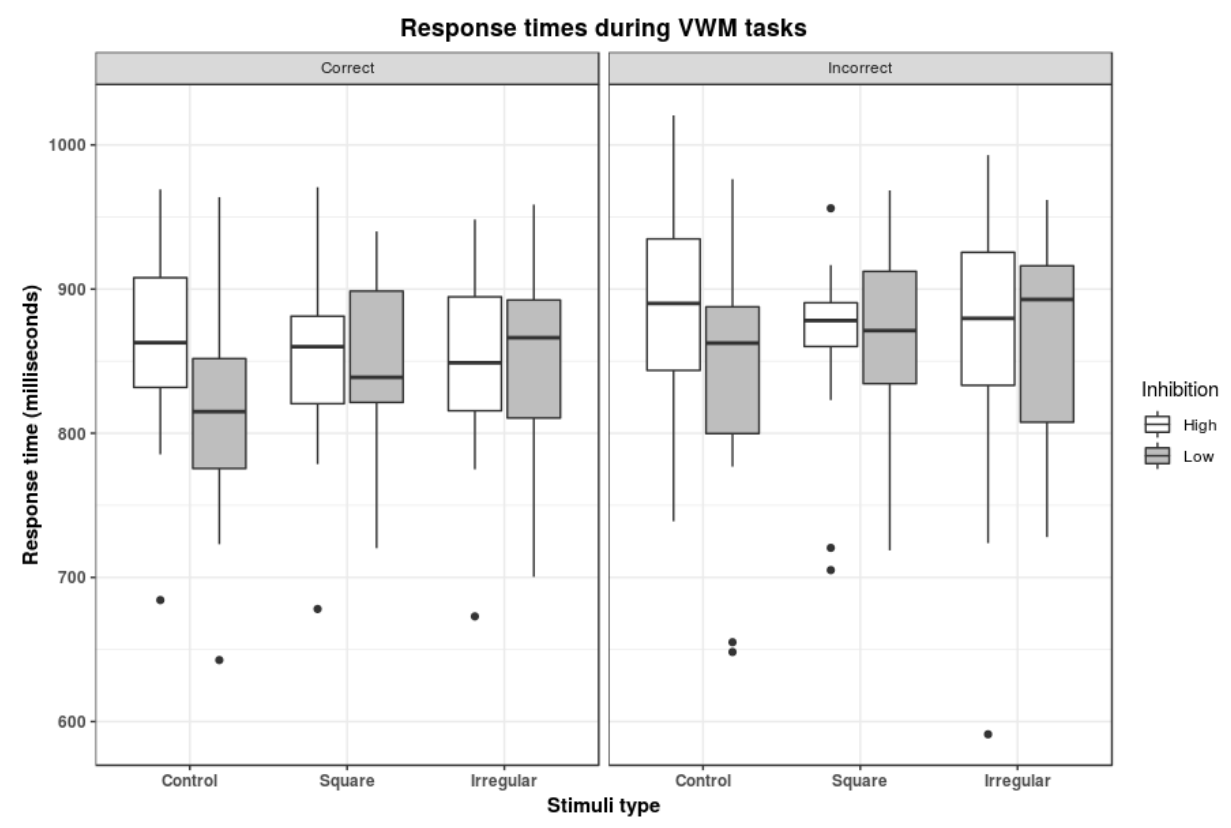

Figure 4. Boxplot displaying the distribution of response times for the VWM task.

Finally, for condition $\gamma$, the distribution $x^{\gamma} \sim B\left(n^{\gamma}, p^{\gamma}\right)$ had the parameters: $n^{\gamma}=2335, p^{\gamma}=$ 0.5062099 Once again, the model with the highest posterior probability was $\theta_{2}=0.5$, with $p \approx 1$. This model's probabilities are shown in figure 7.

With the information obtained from the experiment, we updated the probabilities of our hypothesis in each condition: The probability of $H_{\theta}$ was updated and in the form of the posterior probability $\theta_{2}=0.5$. To update the probability of $H_{1}$, the posterior probabilities of $\theta_{2}=0.6, \theta_{2}=0.7, \theta_{2}=0.8$, and $\theta_{2}=0.9$ were added together. The updated probability of $H_{2}$ was also the sum of $\theta_{2}=0.1, \theta_{2}=0.2, \theta_{2}=0.3$, and $\theta_{2}=$ 0.4 . The new probabilities for the different hypothesis are shown in table 2.

Table 2. Descriptive statistics for response times when exposed to each of the three stimuli types.

\begin{tabular}{llllllllll}
\hline $\begin{array}{l}\text { Response } \\
\text { type }\end{array}$ & $\begin{array}{l}\text { Stimuli } \\
\text { type }\end{array}$ & $\mathrm{n}$ & Mean & SD & Median & MAD & Min & Max & SE \\
\hline Correct & Control & 30.00 & 838.83 & 79.18 & 841.86 & 86.44 & 642.67 & 969.12 & 14.46 \\
& Square & 30.00 & 849.79 & 63.87 & 856.77 & 53.23 & 678.10 & 970.64 & 11.66 \\
& Irregular & 30.00 & 849.65 & 68.17 & 856.05 & 59.07 & 672.96 & 958.60 & 12.45 \\
\hline Incorrect & Control & 30.00 & 862.04 & 88.40 & 870.93 & 80.98 & 648.27 & 1020.50 & 16.14 \\
& Square & 30.00 & 861.98 & 70.89 & 875.67 & 38.67 & 705.06 & 968.46 & 12.94 \\
& Irregular & 30.00 & 862.69 & 87.77 & 886.23 & 80.23 & 591.17 & 992.93 & 16.02 \\
\hline
\end{tabular}

Note: $\mathrm{SD}=$ Standard Deviation, MAD = Median Absolute Deviation, $\mathrm{SE}=$ Standard Error.

Table 3. Response type proportions given Inhibition group and stimuli type.

\begin{tabular}{lllcccc}
\hline Inhibition & Stimuli type & Hit & Miss & FA & CR & No response \\
\hline High & Control & 0.19 & 0.21 & 0.07 & 0.35 & 0.18 \\
& Square & 0.19 & 0.22 & 0.07 & 0.36 & 0.16 \\
& Irregular & 0.18 & 0.22 & 0.06 & 0.35 & 0.19 \\
\hline Low & Control & 0.19 & 0.20 & 0.09 & 0.33 & 0.18 \\
& Square & 0.18 & 0.20 & 0.08 & 0.31 & 0.22 \\
& Irregular & 0.20 & 0.19 & 0.08 & 0.32 & 0.22 \\
\hline
\end{tabular}

Note: $\mathrm{FA}=$ False Alarm, $\mathrm{CR}=$ Correct Rejection. 


\section{Condition $\alpha$}

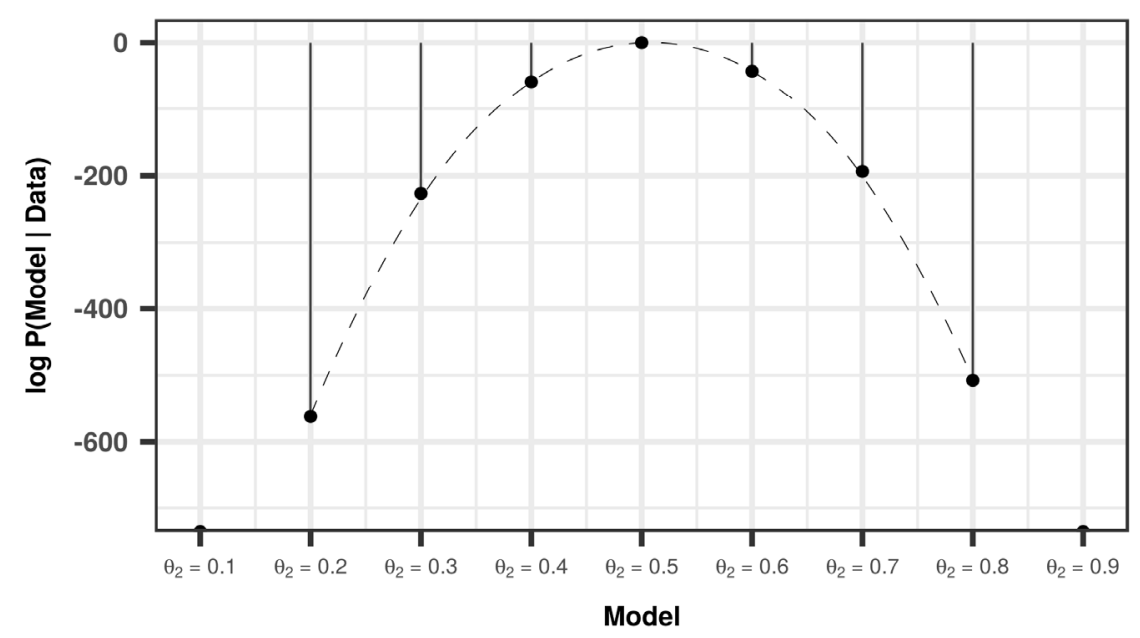

Figure 5. Posterior probability of the models under condition $\alpha$.

Values shown are natural logarithms of the posterior probabilities.

\section{Condition $\beta$}

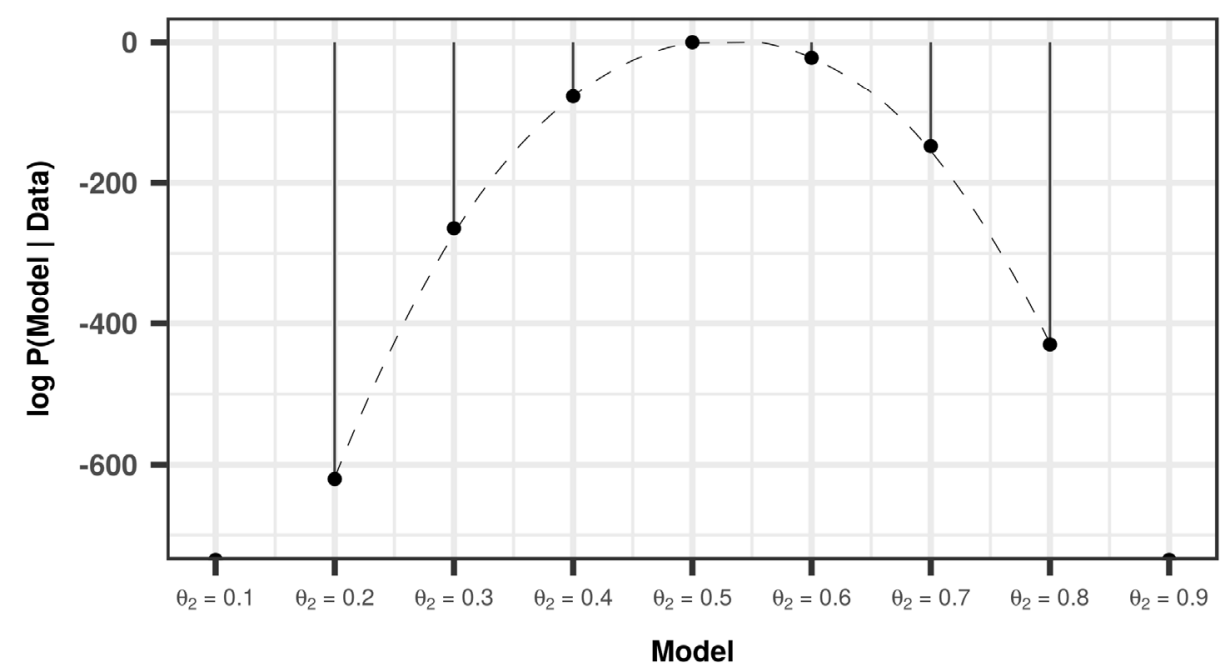

Figure 6. Posterior probability of the models under condition $\beta$.

Values shown are natural logarithms of the posterior probabilities. 


\section{Condition $\gamma$}

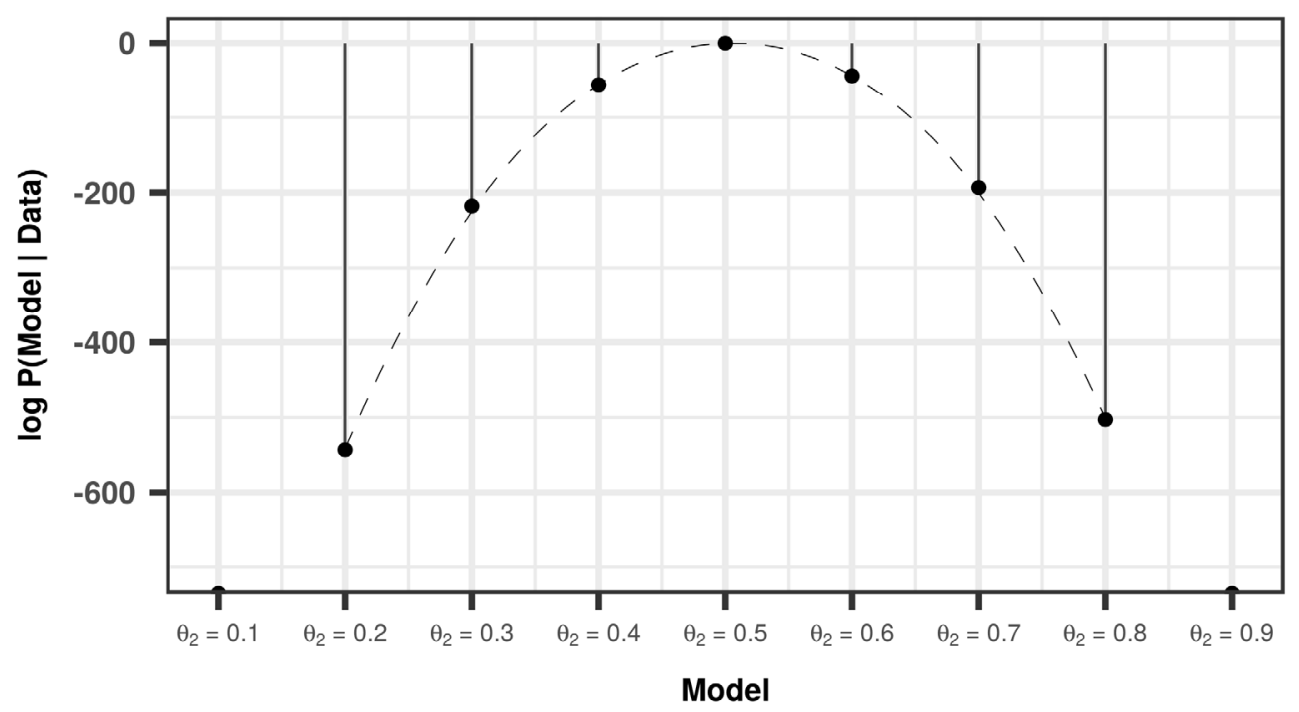

Figure 7. Posterior probability of the models under condition $\gamma$.

Values shown are natural logarithms of the posterior probabilities.

Table 4. Posterior probability of the hypotheses under the different experimental conditions.

\begin{tabular}{cccc}
\hline Condition & Hypothesis & Prior probability & $\begin{array}{c}\text { Posterior } \\
\text { probability }\end{array}$ \\
\hline$\alpha$ & $H_{\theta}$ & 0.5 & $\approx 1$ \\
& $H_{1}$ & 0.25 & $1.724676 \mathrm{e}-18$ \\
& $H_{2}$ & 0.25 & $2.339637 \mathrm{e}-25$ \\
$\beta$ & $H_{\theta}$ & 0.5 & $\approx 1$ \\
& $H_{1}$ & 0.25 & $1.799750 \mathrm{e}-09$ \\
& $H_{2}$ & 0.25 & $1.351169 \mathrm{e}-33$ \\
$\gamma$ & $H_{\theta}$ & 0.5 & $\approx 1$ \\
& $H_{1}$ & 0.25 & $6.564464 \mathrm{e}-19$ \\
& $H_{2}$ & 0.25 & $5.135146 \mathrm{e}-24$ \\
\hline
\end{tabular}

\section{Discussion}

Under all experimental conditions the hypothesis with most plausibility given the data observed during the experiment, was $H_{\theta}$. This indicates the absence of a relationship between inhibitory control and working memory. Our results are in line with those of Redick and collaborators (Redick et al., 2011) .However, these authors studied working memory in its phonetic manifestation, unlike our research. We do not take the attentional account for our results however.

In first place, we assessed young adults, in which clear differences between inhibitory control and WM has been reported (Shing et al., 2010; Wright \& Diamond, 2014). Our results may reflect a difference in cognitive processing developed by ontogeny. Our inhibitory Go/No-go task was made of complex polygons differing in color; and our vWM task was base on complex gray black polygons with an interfering frame. In this context we can account for a lack of influences between processes. This absence of correlation may be related to the nature of stimuli, however.

In second place, it is possible that the relationship between working memory and inhibitory control is dependent on working memory's sensory channel. It is also possible that working memory is related mostly to attentional 
control(Kane, Bleckley, Conway \& Engle, 2001), and in turn, that this capacity itself is not related to inhibitory control for complex visual stimuli, like those used in our study. Regarding the experimental conditions, it has been previously shown that visual stimuli with different characteristics can be clustered together and retained as unitary entities (Luck \& Vogel, 1997). There is also evidence showing that the lesser the amount of entities retained in memory, the bigger the probability of answering correctly in working memory tasks (Cowan, 2001). However, no effect of the experimental manipulation was found in the current research, which may indicates that the effect reported by Luck and Vogel has a limit regarding the area in the visual field in which different entities may be clustered (Luck \& Vogel, 1997). Otherwise, the four polygons we shown to subjects would have been clustered in at least one of the experimental conditions, which in turn would imply a bigger probability of emitting a correct response (Cowan, 2001).

In our study, Go/No-go task could assess motor inhibition (Friedman \& Miyake, 2004), and vWM experimental conditions probably not assessed a motor domain. This interpretation may reflect a stimulus-driven load (remarking items characteristics and spatial position of the target stimuli) for the $\mathrm{vWM}$ task, which probably is a different process from the memory-driven inhibition (recalling the instruction for which to respond or not to the stimuli) in the Go/No-go task. This agree with the results in a recent study by Barret and collaborators (Barrett, Shimozaki, Jensen, \& Zobay, 2016) showing that the relationship between inhibitory control and WM may work together to reduce interfering information and enhance accuracy rates.

In our Bayesian modeling for the relationship between inhibitory control and WM, for both experimental conditions $(\beta, \gamma)$ the hypothesis with greater posterior probability was the null hypothesis $H_{\theta}$. This not necessary mean a rejection to the approach for which inhibitory control influence WM however. We must recall that previous studies have shown that in young adult behavioral data, like those subjects in our study, both cognitive processes memory and inhibition can be distinguished (Shing et al., 2010).

Our results do not lead to accept the WM like an embedded process of a large independent cognitive system however. It may be that we could not confirm a positive relationship between processes because of the variables of our study task. In this case, We are aware that further research that may include visual and auditory modalities of wMT, as well as forms of interactions with inhibitory demands; and cognitive forms of inhibitory control testing should be undertaken to confirm the veracity of our results, by using a Bayesian analysis.

\section{Declarations of interest}

The authors declare that they have no competing interests.

\section{Acknowledgments}

We thank Benemerita Universidad Autónoma de Puebla for it's support via the Research and Posgraduate studies Vice-Rectory (Project Grant: 00313).

\section{References}

Atkinson, R.C., \& Shiffrin, R.M. (1968). Human Memory: A Proposed System and its Control Processes. In In Kenneth W. Spence and Janet Taylor Spence (Ed.), Psychology of Learning and Motivation, volume 2 (pp. 89-195). New York: Academic Press.

Baddeley, A. D. (1992). Working memory. Science, 255(5044), 556-559. https://doi.org/10.1126/science.1736359

Baddeley, A.D. (2003). Working memory: Looking back and looking forward. | Reviews Neuroscience, 4(10), 829-839. https://doi.org/10.1038/nrn1201

Baddeley, A. D.(2017). Exploring working memory. Selected works of Alan Baddeley. Taylor \& Francis Group. https://doi.org/10.4324/9781315111261

Baddeley, A. D., \& Hitch, G. (1974). Working Memory. In G. H. B. T.-P. of L. and M. Bower (Ed.) (Vol. 8, pp. 47-89). Academic Press. https://doi.org/https://doi.org/10.1016/S0079-7421(08)60452-1

Barrett, D. J. K., Shimozaki, S. S., Jensen, S., \& Zobay, O. (2016). Visuospatial working memory mediates inhibitory and facilitatory guidance in preview search. Journal of Experimental Psychology: Human Perception and Performance, 42(10), 1533-1546. https://doi.org/10.1037/xhp0000239

Beaman, C. P. (2010). Working Memory and Working Attention What Could Possibly Evolve? Current Anthropology, 51, S27-S38. https://doi.org/10.1086/650297

Borella, E., Carretti, B., \& De Beni, R. (2008). Working memory and inhibition across the adult life-span. Acta Psychologica, 128(1), 33-44. https://doi.org/10.1016/j.actpsy.2007.09.008 
Carriedo, N., Corral, A., Montoro, P. R., Herrero, L., \& Rucian, M. (2016). Development of the updating executive function: From 7-year-olds to young adults. Developmental Psychology, 52(4), 666-678. https://doi.org/10.1037/dev0000091

Cassel, J.-C., Cassel, D., \& Manning, L. (2013). From Augustine of Hippo's Memory Systems to Our Modern Taxonomy in Cognitive Psychology and Neuroscience of Memory: A 16-Century Nap of Intuition before Light of Evidence. Behavioral Sciences, 3, 21-41. https://doi.org/10.3390/bs3010021

Cowan, N. (1988). Evolving conceptions of memory storage, selective attention, and their mutual constraints within the human information-processing system. Psychological Bulletin, 104(2), 163-191. https://doi.org/10.1037/0033-2909.104.2.163

Cowan, N. (1999). An embedded-processes model of working memory. Models of working memory. In P. Miyake, Akira \& Shah (Ed.), Models of Working Memory: Mechanisms of Active Maintenance and Executive Control (pp. 62-101). New York.

Cowan, N. (2001). The magical number 4 in short term memory. A reconsideration of storage capacity. Behavioral and Brain Sciences, 24(4), 87-186. https://doi.org/10.1017/S0140525X01003922

Dempster, F. N. (1991). Inhibitory processes: a negleted dimension of intelligence. Intelligence, 15(2), 157-173. https://doi.org/10.1016/0160-2896(91)90028-C

Duell, N., Icenogle, G., Silva, K., Chein, J., Steinberg, L., Banich, M. T., ... Chaudhary, N. (2018). A cross-sectional examination of response inhibition and working memory on the Stroop task. Cognitive Development, 47(February), 19-31. https://doi.org/10.1016/j.cogdev.2018.02.003

Engle, R. W. (2002). Working memory capacity as executive attention. Current Directions in Psychological Science, 11(1), 19-23. https://doi.org/10.1111/1467-8721.00160

Friedman, N. P., \& Miyake, A. (2004). The Relations Among Inhibition and Interference Control Functions: A Latent-Variable Analysis. Journal of Experimental Psychology: General, 133(1), 101-135. https://doi.org/10.1037/0096-3445.133.1.101

Gomez, P., \& Perea, M. (2007). A model of the Go/No-Go Task. Journal of Experimental Psychology-General, 136(3), 389-413. https://doi.org/10.1037/0096-3445.136.3.389.A

Iacobucci, D., Posavac, S. S., Kardes, F. R., Schneider, M. J., \& Popovich, D. L. (2015). The median split: Robust, refined, and revived. Journal of Consumer Psychology, 25(4), 690-704. https://doi.org/10.1016/j.jcps.2015.06.014

Kane, M.J., Bleckley, M.K., Conway, A.R.A., \& Engle, R. W. (2001). A controlled-attention view of working-memory capacity. Journal of Experimental Psychology: General, 130(2), 169-183. https://doi.org/10.1037/0096-3445.130.2.169

Luck, S. J., \& Vogel, E. (1997). The capacity of visual working memory for features and conjunctions. Nature, 390(6657), 279-281. https://doi.org/10.1038/36846

Miller, G.A., Galanter, E. \& Pribram, K. H. (1960). Plans and the structure of behavior. Animal Genetics. Holt, Rinehart and Winston, Inc. https://doi.org/10.1037/10039-000

Moffitt, T. E., Arseneault, L., Belsky, D., Dickson, N., Hancox, R. J., Harrington, H., ... Caspi, A. (2011). A gradient of childhood self-control predicts health, wealth, and public safety. Proceedings of the National Academy of Sciences, 108(7), 2693 LP-2698. https://doi.org/10.1073/pnas.1010076108

Redick, T. S., Calvo, A., Gay, C. E., \& Engle, R. W. (2011). Working memory capacity and go/no-go task performance: Selective effects of updating, maintenance, and inhibition. Journal of Experimental Psychology: Learning Memory and Cognition, 37(2), 308-324. https://doi.org/10.1037/a0022216

Repovš, Grega \& Baddeley, A. D. (2006). The multi-component model of working memory: Explorations in experimental cognitive psychology. Neurscoence, 139(1), 5-21. https://doi.org/10.1016/j.neuroscience.2005.12.061

Shing, Y. L., Lindenberger, U., Diamond, A., Li, S.-C., \& Davidson, M. C. (2010). Memory Maintenance and Inhibitory Control Differentiate from Early Childhood to Adolescence. Developmental Neuropsychology, 35(6), 679-697. https://doi.org/10.1080/87565641.2010.508546

Stone, J. V. (2006). Bayes' Rule A Tutorial Introduction to Bayesian Analysis. Understanding Uncertainty. https://doi.org/10.1002/0470055480.ch6 
Team, R. D. C. (2011). R: A Language and Environment for Statistical Computing.le. Viena: the R Foundation for Statistical Computing. Retrieved from http://www.r-project.org/

Wright, A., \& Diamond, A. (2014). An effect of inhibitory load in children while keeping working memory load constant. Frontiers in Psychology, 5(MAR), 1-9. https://doi.org/10.3389/fpsyg.2014.00213

\section{Copyrights}

Copyright for this article is retained by the author(s), with first publication rights granted to the journal.

This is an open-access article distributed under the terms and conditions of the Creative Commons Attribution license (http://creativecommons.org/licenses/by/3.0/). 